\title{
Quantum manifestations of chaos in elastic atom-surface scattering
}

\author{
R. Guantes and S. Miret-Artés \\ Instituto de Matemáticas y Física Fundamental, Consejo Superior de Investigaciones Científicas, Serrano, 123, 28006 Madrid, Spain \\ F. Borondo \\ Departamento de Química, C-IX, Universidad Autónoma de Madrid, Cantoblanco, 28049 Madrid, Spain
}

(Received 17 January 2001; published 10 May 2001)

\begin{abstract}
Quantum manifestations of chaos in the diffraction of atoms from corrugated surfaces, for a range of initial conditions easily attainable in scattering experiments, are presented and discussed. The appearance of strong oscillations in diffraction patterns is shown to be directly related to the presence of classical chaos and threshold effects. We also show that the autocorrelation function for some of the collision $\mathcal{S}$-matrix elements over incident angles is sensitive to the character, hyperbolic or nonhyperbolic, of the underlying chaotic dynamics, in agreement with general semiclassical arguments for unbound chaotic systems.
\end{abstract}

DOI: 10.1103/PhysRevB.63.235401

The identification of universal features that permit one to distinguish between chaotic and regular scattering at the quantum (observable) level is a key issue in recent research in many branches of physics. For instance, there has recently been much interest in the investigation of mesoscopic systems, which are ideal laboratories where quantum manifestations of irregular dynamics can be theoretically studied and experimentally checked. ${ }^{1,2}$ Further recent examples can be found in low-energy electron diffraction experiments, ${ }^{3}$ photodissociation cross sections of molecules, ${ }^{4}$ or cold atoms in accelerated optical lattices, ${ }^{5}$ to mention just a few cases.

Two main approaches have been used to investigate the signature of chaos in quantum systems: one is based on random matrix theory, ${ }^{6}$ and the other on semiclassical arguments ${ }^{7,8}$ expressing observables such as cross sections, transition probabilities, and density of states in terms of classical trajectories. It is now well established that a statistical description based on the first formalism, in analogy with bounded systems, is also applicable to scattering problems by using ensembles appropriate to the scattering $\mathcal{S}$ matrix, which is non-Hermitian and unitary. ${ }^{6}$ In this work and within the second approach, we present some evidence for different manifestations of classical chaos in elastic atom-surface diffraction intensities within a range of initial conditions [incident energies between 7 and $100 \mathrm{meV}$, and polar angles, measured from the normal $z$ axis to the surface taken to be placed in the $(x, y)$ plane, between $0^{\circ}$ and $\left.90^{\circ}\right]$ that are easily attainable in experiment.

As a working example, we choose to study the scattering of He atoms from nearly one-dimensional (say in the $x$ direction, perpendicular to the step edge) corrugated $\mathrm{Cu}(11 \alpha)$ surfaces, with $\alpha=0,3,5,7$, which has been extensively studied both experimentally ${ }^{9}$ and theoretically within a quantal and semiclassical ${ }^{10}$ framework. From experimental diffraction patterns at different incident conditions, a two degrees of freedom $(x, z)$, periodic, and corrugated Morse potential ${ }^{9}$ was fitted, and it has been widely used in all of the calculations mentioned above. It essentially consists of a Fourier series with a Morse potential in the $z$ direction and three more terms describing the corrugation (or roughness) of the surface in the $x$ direction as well as the couplings. In particular, for $\alpha=5,7$, the coupling is strong enough to produce
PACS number(s): 05.45.Mt, 79.20.Rf

observable features associated with resonances (angular widths greater than $5^{\circ}$ versus typical angular experimental resolutions of about $0.5^{\circ}$ ) and thresholds. In parallel work, the classical scattering for these systems has been shown to display chaos as a function of the incident angle and energy. ${ }^{10}$ As these parameters are varied, the onset of classical chaos occurs when one surface rainbow (a maximum or minimum of the final scattering angle as a function of the impact parameter on the surface) reaches the value $\pm \pi / 2$. This condition implies that the particle leaves the interaction region traveling parallel to the surface. Past this value, the particle experiences more than one collision (temporary classical trapping), losing memory of the initial conditions, which is the origin of chaos in this problem. We have also investigated how the bound component of the dynamics (periodic orbits) influences the scattering trajectories and becomes more chaotic as the incident energy decreases, ${ }^{11}$ the number of open (or energetically accessible) diffraction channels being smaller. This result is in sharp contrast to what usually happens in bounded systems.

When elastic diffraction patterns at fixed beam energies are calculated, several features are clearly visible (and observable) due mainly to two pure quantal effects: the socalled threshold resonances (TR's) and selective adsorption resonances (SAR's). Threshold resonances appear when a diffraction channel or beam just becomes visible or evanescent, and correspond to very general features of any scattering system. In constrast, SAR's occur when the scattering particle is traveling parallel to the surface in free motion but its perpendicular motion is bounded and equal to a bound level of the laterally or surface averaged interaction potential. ${ }^{12}$ The denomination "TR" is somewhat misleading since we are not dealing with true resonances or quasibound states as in SAR's. The corresponding resonance positions in both cases can be easily extracted from kinematic arguments. Thus, for any in-plane $(x, z)$ scattering event, the energy and parallel momentum conservation rules can be globally expressed in terms of the incident wave vector $\mathbf{k}_{i}$ as

$$
\mathbf{k}_{\mathbf{G}, z}^{2}=\mathbf{k}_{i}^{2}-\left(\mathbf{K}_{i}+\mathbf{G}\right)^{2} .
$$

Throughout this work, square wave vectors are given in energy units $\left(\hbar^{2} / 2 \mu=1, \mu\right.$ being the mass of the projectiles), 


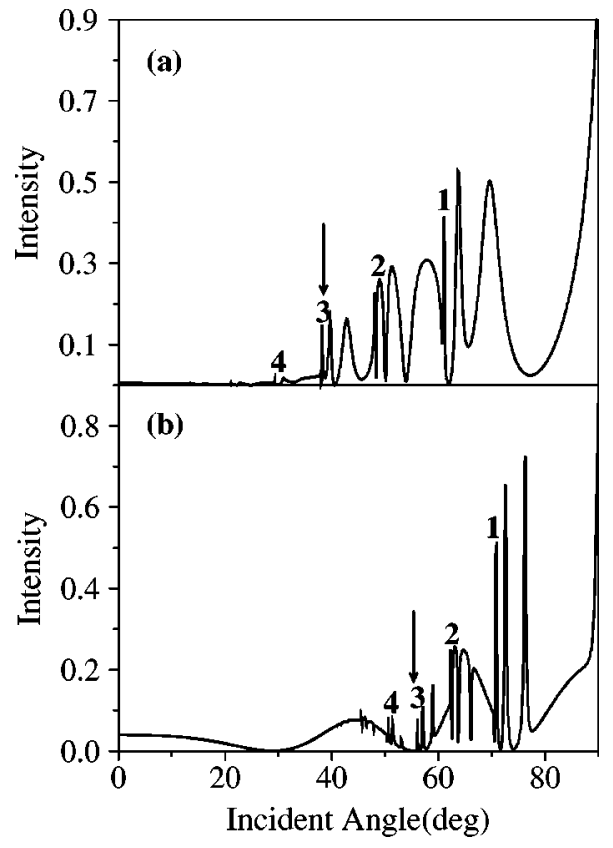

FIG. 1. Specular $[\mathbf{0}=(0,0)]$ intensity versus the incident angle for the ${ }^{4} \mathrm{He}-\mathrm{Cu}(117)$ system, at two different incident energies (a) $15 \mathrm{meV}$ and (b) $75 \mathrm{meV}$. The onset of classical chaos with increasing incident angle is marked with arrows. Numbers indicate the threshold resonances (see text).

vectors parallel to the surface having only the $x$ component are written in capital letters, and vectors in two dimensions in small letters, according to the standard notation; $\mathbf{k}_{\mathbf{G}, z}$ is the $z$ component of the final wave vector, $\mathbf{G}$ is a general reciprocal lattice vector, and $\mathbf{K}_{i}=k_{i} \sin \theta_{i}$ is the parallel incident wave vector $\left(\theta_{i}\right.$ being the incident angle). The following kinematic conditions have to be fulfilled for the two kinds of resonance:

(i) The TR condition,

$$
\mathbf{k}_{\mathbf{N}, z}^{2}=\mathbf{k}_{i}^{2} \cos ^{2} \theta_{f}=0
$$

where $\mathbf{N} \in \mathbf{G}$ is the reciprocal lattice vector associated with an emerging or evanescent diffraction channel, $\theta_{f}= \pm \pi / 2$ being a Bragg (and therefore observable) final scattering angle. Threshold angles are those incident angles leading to such Bragg final scattering angles.

(ii) The SAR condition,

$$
\mathbf{k}_{\mathbf{F}, z}^{2}=\mathbf{k}_{i}^{2}-\left(\mathbf{K}_{i}+\mathbf{F}\right)^{2}=-\left|\epsilon_{n}\right|,
$$

where $\mathbf{F} \in \mathbf{G}$ is the reciprocal lattice vector exchanged in the resonance process (in this case a closed, or not energetically accessible, channel) and $\epsilon_{n}$ is the $n$th bound state of the attractive, surface averaged Morse potential resulting in the $z$ direction.

In Fig. 1, quantal close-coupling specular $[\mathbf{0}=(0,0)]$ intensities versus incident angle for the $\mathrm{He}-\mathrm{Cu}(117)$ system at two incident energies of 15 and $75 \mathrm{meV}$ are plotted. After a TR (labeled by an integer number), a series of oscillations attributed to SAR's displaying Lorentzian or Fano-type line shapes are clearly visible. Thus, for example, at $15 \mathrm{meV}$ and between $60^{\circ}$ and $90^{\circ}$ [Fig. 1(a)], the closed diffraction chan- nel associated with the $\mathbf{F}=(1,0)$ reciprocal lattice vector is the one exchanged in the resonance process, the oscillations being due to the existence of four bound states supported by the Morse potential. At that low energy, however, the overlapping of SAR's coming from different close $\mathbf{F}$ channels such as the $\mathbf{F}=(1,0)$ and $\mathbf{F}=(2,0)$ vectors takes place. Moreover, resonances corresponding to higher bound states accumulate at the TR feature and their angular widths decrease gradually. With decreasing $\theta_{i}$, the successive channels associated with $(2,0),(3,0)$, and $(4,0)$ play the role of exchanged reciprocal lattice vectors in the resonance process until each one becomes a TR, which has been labeled by $\mathbf{N}$ $=2,3,4$, respectively. A similar pattern is reproduced around all the different threshold angles. Analogously, for $75 \mathrm{meV}$ [Fig. 1(b)], we observe the same structure of TR and SAR series but with two important differences: the angular widths are smaller here and SAR's coming from different close channels do not overlap. However, in both cases, a clear transition in the intensity of the oscillations occurs very near to the onset of classical chaos, which is marked with arrows in Fig. 1. The global intensity behavior, except for the onset of large fluctuations, can be qualitatively interpreted in a multichannel scattering framework. The relative arrangement of the diffraction channels in energy as well as their effective couplings to the entrance channel are responsible for the interference between the background and resonant contributions to the $\mathcal{S}$ matrix. In particular, the coupling of the specular channel to the rest of the channels decreases with increasing diffraction order and therefore the intensity gradually increases with $\theta_{i}$. As has been previously done in other contexts, the underlying chaotic dynamics found in this scattering will be invoked to provide an alternative and more fundamental interpretation.

In order to explain the features described above, we start with the semiclassical expression for the $\mathcal{S}$-matrix elements deduced by Miller: ${ }^{14}$

$$
\mathcal{S}_{\mathbf{0 G}}\left(E, \theta_{i}\right)=\sum_{s}\left|P_{\mathbf{0 G}}^{(s)}\right|^{1 / 2} \exp \left(\frac{i}{\hbar} S^{(s)}-i \frac{\pi}{2} \mu_{s}\right),
$$

where $P_{\mathbf{0 G}}^{(s)}$ is the classical probability for a trajectory $s$ to end up with a final momentum in the parallel direction $p_{x_{f}}=p_{x_{i}}$ $+2 \pi \hbar m / a$, with $\mathbf{G}=(m, 0)$ (Bragg diffractin condition); and $S^{(s)}$ is the corresponding reduced classical action depending only on the variables $p_{x_{i}}$ and $p_{x_{f}}$. The general intensity behavior of a diffraction pattern (the envelope of the diffraction intensities) can be extracted from the classical transition probabilities, including interferences. In this respect, an analysis of the classical singularities will provide the main qualitative features of the intensity envelope. These singularities occur when the amplitude in Eq. (4) goes to infinity. In the semiclassical theory, $P_{\mathbf{0 G}}^{(s)}=|\partial G(b) / \partial b|^{-1}$ evaluated at $b=b_{s}$, where $G(b)=a \sqrt{2 \mu E_{i}}\left[\sin \theta_{f}(b)\right.$ $\left.-\sin \theta_{i}\right] / 2 \pi \hbar$, is the so-called classical diffraction order function and $b$ is the impact parameter normalized to the unit cell length $a$. Therefore one finds that, apart from the well known rainbow singularity occurring when $d \theta_{f}(b) / d b=0$, another singularity (the so-called skipping singularity) 


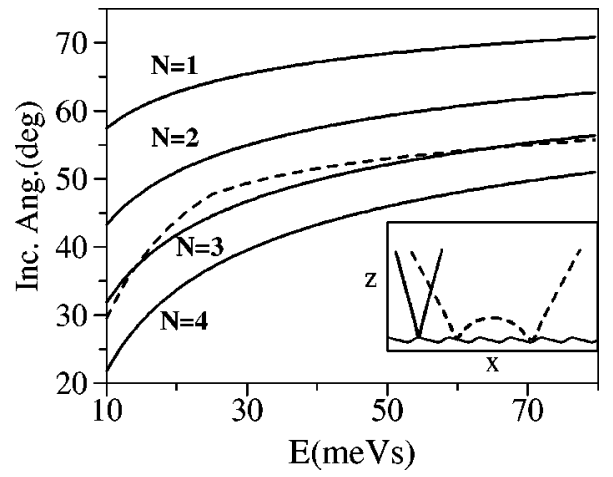

FIG. 2. Onset of chaos as a function of incident conditions in energy and angle for the ${ }^{4} \mathrm{He}-\mathrm{Cu}(117)$ system (dashed line). The solid lines are the threshold resonance conditions for the channels $\mathbf{N}=(1,0), \ldots,(4,0)$. In the inset two typical scattering trajectories at $15 \mathrm{meV}$ are plotted: with a solid line, a direct trajectory in the regular regime $\left(\theta_{i}=29^{\circ}\right)$, and with a dashed line, a chaotic trajectory above the onset of chaos $\left(\theta_{i}=48^{\circ}\right)$. The equipotential line at that energy is also plotted.

appears ${ }^{13}$ whenever the classical deflection function $\theta_{f}(b)$ reaches $\pm \pi / 2$ (this angle being not necessarily a Bragg angle), which implies a condition for trapping of trajectories or classical chaos as noted above. When $\theta_{f}(b)= \pm \pi / 2$ coincides with a Bragg angle, this classical singularity also matches the TR condition as defined by Eq. (2), and one would expect a change in the intensity pattern.

In Fig. 2, the onset of classical chaos and some TR conditions, Eq. (2), as a function of the incident energy and angle in the ranges usually accessible by experiment are plotted. As an illustration, in the inset of the figure, at an incident energy of $15 \mathrm{meV}$, two typical trajectories contributing to the semiclassical specular intensities according to Eq. (4) are also shown: one in the regular region at $\theta_{i}=29^{\circ}$, and the other in the chaotic region (trapped trajectories), at $\theta_{i}$ $=48^{\circ}$. Due to the nonlinear dynamical analysis, two types of threshold can be distinguished depending on relative position with respect to the onset of classical chaos: TR's appearing in the regular regime $(\mathbf{N}=4)$ and those in the chaotic regime $(\mathbf{N}=1,2)$. Interestingly enough, the threshold $\mathbf{N}=3$ appears around the onset of chaos and also changes the intensity behavior as shown in Fig. 1. In the first case, the TR channel $\mathbf{N}$ is energetically allowed but dynamically forbidden (there are no real classical trajectories fulfilling the condition $\theta_{f}=$ $\pm \pi / 2$ ). From a semiclassical point of view, only direct trajectories would contribute to the specular intensity. In the second case, the $\mathbf{N}$ channel is both dynamically and energetically allowed, chaos will set in, and an infinity of real chaotic trajectories interfering with direct trajectories will contribute to the specular intensity.

The influence of the nonlinear underlying dynamics can be studied through the correlation functions of some $\mathcal{S}$-matrix element such as, for example, the specular one written as

$$
C_{E_{i}}(\phi)=\left\langle\mathcal{S}_{00}^{*}\left(E_{i}, \theta_{i}\right) \mathcal{S}_{00}\left(E_{i}, \theta_{i}+\phi\right)\right\rangle_{\theta_{i}},
$$

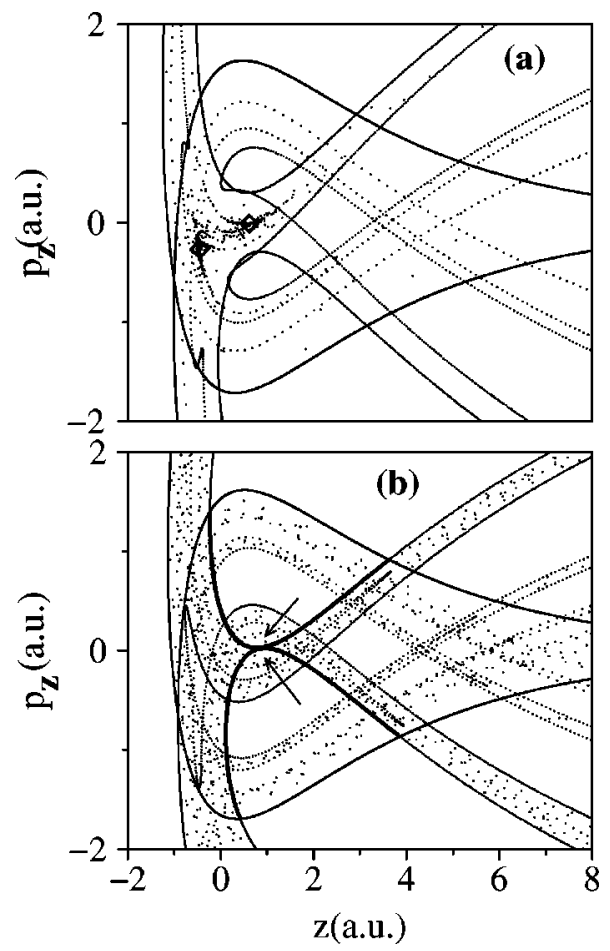

FIG. 3. Poincare surface of section at $x=n a$ of the homoclinic tangle generated from the parallel free asymptotic $\mathrm{He}$ atom motion at two different energies: (a) $75 \mathrm{meV}$, where a period 2 stable periodic orbit (fixed points with diamonds) exists, causing sticking of nearby trajectories; and (b) $47 \mathrm{meV}$, where the last homoclinic tangency between the entrance and exit lobes (thick solid lines) takes place and is marked with arrows in the figure.

where $\phi$ is the incident angle difference and the average is over a range of initial angles in the chaotic region greater than $\phi$. A similar approach was followed by Lai et al. ${ }^{17}$ and other authors ${ }^{1,2}$ who showed that spectral and transport properties behave quite differently depending on the nature of the classical dynamics. The incident energies of 15 and $75 \mathrm{meV}$ have been chosen because they are representative of two different types of classical regime: hyperbolic and nonhyperbolic, respectively. The transition between them can be obtained by calculating the homoclinic tangle generated from the parallel free asymptotic He atom motion at different energies. In Fig. 3 we show the corresponding Poincaré surface of section at two energies. At high energies, stable periodic orbits and cantori existing in the interaction region [Fig. $3(\mathrm{a})$ ] cause long term trapping of the trajectories close to them. This causes the escape rate to decay algebraically for long time. ${ }^{15}$ At an energy of approximately $47 \mathrm{meV}$, a chaotic transition takes place when the stable and unstable manifolds of the parallel He motion intersect with each other for the last time [the last homoclinic tangency, ${ }^{16}$ marked with arrows in Fig. 3(b)]. Below this energy, no islands of stability survive and the fingerprint of this hyperbolic regime is an exponential decay in the escape rate. ${ }^{8}$

Using again the semiclassical approximation to the $\mathcal{S}$-matrix elements Eq. (4), we expand the classical action to first order in $\phi$. The chain rule can be used to express the derivative with respect to $\theta_{i}$ in terms of derivatives with 


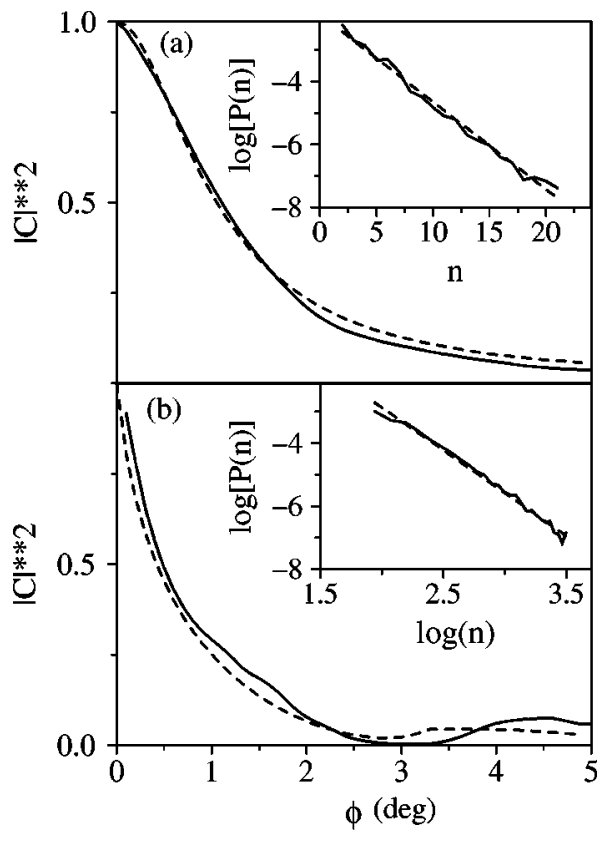

FIG. 4. Quantum (solid lines) and semiclassical (dashed lines) correlation functions of the specular $\mathcal{S}$-matrix elements, Eqs. (5) and (6), at energies (a) $15 \mathrm{meV}$ and (b) $75 \mathrm{meV}$. The insets represent the classical unit cell distribution functions (averaged in angle) at the energies indicated, together with the analytical fittings (dashed lines).

respect to $p_{x_{i}}$ and $p_{x_{f}}$. In the asymptotic region, these derivatives give the net distance in $x$ traveled without interaction, so that $S^{(s)}\left(\theta_{i}+\phi\right)=S^{(s)}\left(\theta_{i}\right)+\phi p\left(x_{f}-x_{i}\right) \cos \theta_{i}, p$ being the modulus of the momentum. Finally, the exponent can be cast in terms of $\phi$ and the number of unit cells traveled by the trajectory $s$ in the interaction region, $n=\left(x_{f}-x_{i}\right) / a$. In doing so, we can label the trajectories $s$ in Eq. (4) by the number of unit cells (or Poincaré recurrences taking as a surface of section $\left.x=x_{i}+n a\right)$ traveled during the interaction time. Substituting this into the expression for $C_{E_{i}}(\phi)$ and neglecting cross term contributions, one obtains

$$
C_{E_{i}}(\phi) \sim \sum_{n} P(n)\left\langle e^{i \phi \beta n}\right\rangle_{\theta_{i}}
$$

where $P(n)$ is the classical probability for a trajectory to jump $n$ unit cells in the interaction region (independent of the incident angle) and $\beta=a k_{i} \cos \theta_{i}$. The behavior of the unit cell distribution probability $P(n)$ is identical to that of the escape rate. ${ }^{7}$

The squared moduli of the quantum correlation functions corresponding to the energies shown in Fig. 1 are depicted in
Fig. 4 with solid lines [normalized to unity for $\left.C_{E_{i}}(0)\right]$. The decay rule is slower (and different) in the hyperbolic regime [Fig. 4(a), $15 \mathrm{meV}]$ than in the nonhyperbolic one [Fig. 4(b), $75 \mathrm{meV}$. The corresponding distribution probabilities $P(n)$ are shown in the inset of each figure. At $15 \mathrm{meV}$ no trace of regularity in the interaction region is left and $P(n) \sim e^{-\gamma n}$, with $\gamma \sim 0.3$. An analytical estimation for the shape of the autocorrelation function can be obtained by expanding the exponential dependence of $\phi$, in Eq. (6), in a series of integer order Bessel functions up to the second order (valid for small $\phi$ ). If we replace the discrete sum by an integral in the $n$ variable, this integral is solved analytically and the squared modulus of the correlation function gives a Lorentzian function with the width depending on $\gamma$, in agreement with previous results found for other systems. ${ }^{1,17}$ In Fig. 4(a), the semiclassical result coming from Eq. (6) is depicted with dashed lines; it is calculated numerically with the estimation for $\gamma$ taken from the exponential fitting to $P(n)$, showing a fairly good agreement with the quantum result. On the other hand, at $75 \mathrm{meV}$, two small stability islands coming from a period 2 periodic orbit in the interaction region [Fig. 3(a)] are still found. At long $n$ (for $n>8$ ), we observe an algebraic distribution $P(n) \sim n^{-\alpha}$ with $\alpha \sim 2.5$ [inset of Fig. 4(b)]. This exponent is greater than the usual ones reported for other systems but it is in agreement with a recent analysis of the asymptotic statistics of Poincaré recurrences ${ }^{18}$ whose distribution decays at very long times as $P(\tau) \sim 1 / \tau^{3}$. In our case, we note that the quantum correlation function follows the semiclassical prediction for a slightly lower exponent, $\alpha \sim 2.1$ [dashed line in Fig. 4(b)]. This discrepancy could be attributed to several factors. Among them we want to stress the possibility that, for large $n$, it is numerically difficult to obtain good statistics for $P(n)$, or that quantum mechanics follows the classical predictions mainly for short time scales (short $n) .{ }^{19}$

In conclusion, we have presented a study on quantum diffraction intensities for the $\mathrm{He}-\mathrm{Cu}(117)$ system at incident conditions for which classical chaos is dominant. The main result of our research is that the quantum manifestations of this chaotic behavior can be explained by semiclassical arguments and experimentally detected because the strong oscillations exhibited by the specular peak should be easily observable with the actual angular resolution. The surface temperature should be below $100 \mathrm{~K}$ in order to reduce inelastic effects. A complete, comparative study (classical, semiclassical, and quantal) of the elastic atom-surface scattering dynamics has been carried out.

This work was supported by DGES (Spain) under Contract Nos. PB95-71 and PB98-115 and the European Contract No. HPRN-CT-1999-00005. R.G. acknowledges financial support from CAM (Spain).
${ }^{1}$ H.U. Baranger et al., Chaos 3, 665 (1993); C.M. Marcus et al., ibid. 3, 643 (1993); R. Fleischmann, T. Geisel, and R. Ketzmerick, Phys. Rev. Lett. 68, 1367 (1992).

${ }^{2}$ A.S. Sachrajda et al., Phys. Rev. Lett. 80, 1948 (1998).
${ }^{3}$ P.L. de Andres and J.A. Vergés, Phys. Rev. Lett. 80, 980 (1998).

${ }^{4}$ Y.V. Fyodorov and Y. Alhassid, Phys. Rev. A 58, R3375 (1998).

${ }^{5}$ Q. Niu and M.G. Raizen, Phys. Rev. Lett. 80, 3491 (1998); M. Glück, A.R. Kolovsky, and H.J. Korsch, ibid. 83, 891 (1999). 
${ }^{6}$ C. Jung and T.H. Seligman, Phys. Rep. 285, 77 (1997); C.H. Lewenkopf and H.A. Weidenmüller, Ann. Phys. (N.Y.) 212, 53 (1991).

${ }^{7}$ U. Smilansky, in Chaos and Quantum Physics, edited by M.-J. Giannoni, A. Voros, and J. Zinn-Justin (North-Holland, New York, 1991).

${ }^{8}$ P. Gaspard and S.A. Rice, J. Chem. Phys. 90, 2225 (1989); 90, 2242 (1989); 90, 2255 (1989).

${ }^{9}$ D. Gorse, B. Salanon, F. Fabre, A. Kara, J. Perreau, G. Armand, and J. Lapujoulade, Surf. Sci. 147, 611 (1984); S. Miret-Artés, J.P. Toennies, and G. Witte, Phys. Rev. B 54, 5881 (1996).

${ }^{10}$ R. Guantes, F. Borondo, C. Jaffé, and S. Miret-Artés, Phys. Rev. B 53, 14117 (1996); F. Borondo, C. Jaffé, and S. Miret-Artés, Surf. Sci. 371, 211 (1994).

${ }^{11}$ R. Guantes, F. Borondo, and S. Miret-Artés, Phys. Rev. E 56, 378
(1997).

${ }^{12}$ J.E. Lennard-Jones and A.F. Devonshire, Nature (London) 137, 1069 (1936).

${ }^{13}$ R. Guantes, F. Borondo, J. Margalef-Roig, S. Miret-Artés, and J.R. Manson, Surf. Sci. 375, L379 (1997).

${ }^{14}$ W.H. Miller, Adv. Chem. Phys. 30, 77 (1975).

${ }^{15}$ J.D. Meiss and E. Ott, Physica D 20, 387 (1986).

${ }^{16} \mathrm{~S}$. Wiggins, Introduction to Applied Non Linear Dynamical Systems and Chaos (Springer-Verlag, New York, 1990).

${ }^{17}$ Y.C. Lai, R. Blümel, E. Ott, and C. Grebogi, Phys. Rev. Lett. 68, 3491 (1992).

${ }^{18}$ B.V. Chirikov and D.L. Shepelyansky, Phys. Rev. Lett. 82, 528 (1999).

${ }^{19}$ G. Casati, G. Maspero, and D.L. Shepelyansky, Phys. Rev. Lett. 82, 524 (1999). 